\title{
Giant noise amplification in Synchronously Pumped Optical Parametric Oscillators
}

\author{
G. D'Alessandro and C. Brent Laforet \\ School of Mathematics, University of Southampton, Highfield, Southampton SO17 1BJ, \\ England, UK
}

\begin{abstract}
We show numerically that a synchronously pumped optical parametric oscillator can show giant noise amplification of the order of $10^{9}$. We use pseudospectra to identify the parameter region for giant noise amplification and to estimate its magnitude. (c) 2008 Optical Society of America
\end{abstract}

OCIS codes: $190.4410,190.4970,190.3100,270.2500$

Noise plays a very important role in synchronously pumped optical parametric oscillators (SPOPOs). For example, these devices (see [1] for a description) have been suggested as tunable source of short pulses [2-4]. However, in Ref. [1] it was shown that in the pulse compression regime the signal pulses may suffer from considerable noise-induced jitter. In the context of quantum optics, optical parametric oscillators have been suggested as a tool to amplify and detect quantum fluctuations [5]. However, this requires very large amplification factors.

In order to make full use of SPOPOs for either of these applications, we need to quantify noise amplification. This will allow us to optimize device parameters in order to achieve, for example, cleanest pulse compression or maximal amplification of quantum fluctuations. In this letter we introduce the SPOPO pseudospectrum [6] as a tool to quantify noise amplification. We show that this can be of the order of $10^{9}$ in a sub-threshold SPOPO under standard operating conditions. This value is so high that a noise-driven, non-zero signal field appears even for sub-threshold pump values.

This phenomenon is considerably different from non-resonant optical parametric amplification because the presence of the cavity introduces feedback in the system. This, by itself, is unable to sustain a non-zero signal field. However, in the presence of noise amplification, the feedback allows the signal to develop in a form that is optimal for energy extraction from the pump. In some respects the output is similar to that of an above threshold SPOPO. For example, the power spectrum of the noise-driven signal field is double peaked and asymmetric as is the case for standard SPOPO pulses [7]. On the other hand, the signal field reported 
here is very different from the standard above threshold SPOPO output: firstly, should noise be switched off, the signal field would rapidly decay to zero, i.e. to the stable solution; secondly, if the pump is not saturated, then the amplitude of the signal is proportional to that of the noise.

Giant noise amplification is not restricted to optics [8], where it is often called "excess noise" $[6,9,10]$ : it is, for example, used to explain hydrodynamic turbulence in a laminar flow $[11]$.

The structure of this paper is as follows: we first introduce a standard SPOPO model and define the pseudo-spectrum of a linear operator (matrix). We then combine the two to measure the noise amplification factor for a few representative values of the parameters.

The SPOPO equations for plane wave fields in suitable dimensionless variables are [1]:

$$
\begin{aligned}
& \partial_{z} E_{1}=-v_{1}^{-1} \partial_{t} E_{1}-\left(\rho_{1}-i \Delta k\right) E_{1}+i \beta_{1} \partial_{t t} E_{1}-E_{2} E_{3}, \\
& \partial_{z} E_{2}=-v_{2}^{-1} \partial_{t} E_{2}-\rho_{2} E_{2}+i \beta_{2} \partial_{t t} E_{2}+E_{1} \bar{E}_{3}, \\
& \partial_{z} E_{3}=-v_{3}^{-1} \partial_{t} E_{3}-\rho_{3} E_{3}+i \beta_{3} \partial_{t t} E_{3}+E_{1} \bar{E}_{2},
\end{aligned}
$$

where $E_{j}, j=1,2,3$ are respectively the pump, signal and idler amplitudes, $v_{j}$ are their group velocities, $\beta_{j}$ their dispersion coefficients, $\rho_{j}$ their losses during propagation. $\Delta k$ is the phase mismatch between the three fields. The over-bar symbol denotes complex conjugation. In these equations the coordinate along the cavity, $z$, is scaled to the crystal length, while time is scaled to the time taken by the signal field to cross the crystal; in these units $v_{2}=1$. The fields at the entrance of the crystal are

$$
\begin{aligned}
& E_{1}(0, t)=P(t), \quad E_{3}(0, t)=0, \\
& E_{2}(0, t)=\exp (-i \theta) \sqrt{R} E_{2}\left(1, t-T_{c}+1\right),
\end{aligned}
$$

where $P(t)=P_{a} \exp \left(-t^{2} / \tau_{p}^{2}\right)$ is a Gaussian pump profile of amplitude $P_{a}$ and width $\tau_{p}$. We assume that the pump profile is periodic with period $T_{R}, P(t)=P\left(t+T_{R}\right)$, with $\tau_{p} \ll T_{R}$. $T_{c}$ is the cavity round-trip time for the signal field, $R$ is the total intensity reflection coefficient of the cavity and $\theta$ is the phase shift acquired per pass by the signal field. These equations admit a zero signal and idler solution: $E_{2}(z, t)=E_{3}(z, t) \equiv 0$, while the pump is broadened by dispersion as it propagates through the crystal, but is otherwise unaltered.

It is convenient to study the stability of this solution by recasting equations $(1,2)$ as a map. We indicate with $E_{2}^{(n)}(z, \tau)$ the signal field at the $n$-th pump pulse (approximately the $n$-th cavity round-trip), i.e. at time $t=n T_{R}-\tau$. Equations (1,2) can be written as a relation (map) between signal pulses at successive round-trips: $E_{2}^{(n+1)}(z, \tau)=\mathcal{N}\left[E_{2}^{(n)}\right]$, where $\mathcal{N}$ is a suitable nonlinear operator that depends, in particular, on the pump amplitude $P_{a}$ and the detuning between cavity and pump periods, $\tau_{c} \equiv T_{R}-T_{c}$. The zero signal and idler solution of equations (1) is equivalent to $E_{2}^{(n)}(z, \tau) \equiv 0$ for all values of the round-trip number $n$. 
Small perturbations $e_{2}^{(n)}$ of this solution evolve from one round-trip to the next according to the linear map $e_{2}^{(n)}=\mathcal{L} e_{2}^{(n-1)}$, where $\mathcal{L}$ is a linear operator (ultimately a matrix) obtained by linearizing $\mathcal{N}$ around $E_{2}^{(n)} \equiv 0$. The zero signal and idler solution is stable if all the eigenvalues of $\mathcal{L}$ have modulus less than one. We indicate with $\lambda_{M}$ the largest modulus: the zero signal and idler solution is stable if $\lambda_{M}<1$.

We expect that the signal may be noise-sensitive for sufficiently negative values of $\tau_{c}$, even in the case when $E_{2}^{(n)} \equiv 0$ is stable (see Figure 1). If the pump repetition time is shorter than the cavity round trip time, the (noisy) leading edge of the signal pulse is amplified because it arrives at the crystal when the pump field and, hence, the available gain are very large. On the other hand, the main part of the signal pulse arrives at the crystal when the pump pulse is waning and thus sees little amplification, but only the losses occurred during propagation through the cavity: it decays to zero because the system is below threshold. The new signal pulse is the result of the giant amplification of the noisy leading edge of the previous signal pulse: its dynamics is entirely noise-driven. For positive values of $\tau_{c}$ the signal arrives when the pump is waning and relatively little amplification can be expected. This rough explanation does not take into account the role of the group velocities and of the other OPO parameters. It is possible that large amplification can be seen in other parameter regimes.

The tool that allows us to quantify this description is the pseudospectrum of $\mathcal{L}$. We introduce this concept by first discussing the properties of linear maps. Consider the scalar map $x^{(n+1)}=r x^{(n)}$, with all the $x^{(n)}$ and $r$ real numbers. If $|r|<1$ then $\left|x^{(n)}\right|$ form a decreasing sequence. Now consider the SPOPO map $e_{2}^{(n)}=\mathcal{L} e_{2}^{(n-1)}$ : we could expect that the norm of perturbation $e^{(n)}$ would decrease monotonically if $\lambda_{M}<1$. However, while it is true that the perturbation will asymptotically decay to zero if $\lambda_{M}<1$, it is not true that the decay is necessarily monotonic: in other words, the perturbation can first grow and only at a later stage decay. If the growth is sufficiently large and there is continuous injection of noise, macroscopic noise-driven structures may appear. It can be shown [6] that the maximum growth possible is related to the $\epsilon$-pseudospectrum (or pseudospectrum) of $\mathcal{L}$. This is defined for any real number $\epsilon>0$ as $[6]$

$$
\Lambda_{\epsilon}(\mathcal{L})=\left\{z \in \mathbb{C}:\left\|(z-\mathcal{L})^{-1}\right\| \geq \epsilon^{-1}\right\}
$$

For $\epsilon=0$ this is just the standard spectrum; for $\epsilon>0, \Lambda_{\epsilon}$ consists of patches of the complex plane that surround the eigenvalues of $\mathcal{L}$, i.e. contain its spectrum. The boundary of $\Lambda_{\epsilon}$ gives graphical and quantitative information on the maximum growth possible. This is at least equal to Kreiss constant [6],

$$
\mathcal{K}=\sup _{\epsilon>0} \mathcal{K}_{\epsilon}, \quad \text { with } \quad \mathcal{K}_{\epsilon} \equiv \frac{\rho_{\epsilon}(\mathcal{L})-1}{\epsilon},
$$


where $\rho_{\epsilon}(\mathcal{L})$ is the radius of the smallest circle that contains $\Lambda_{\epsilon}(\mathcal{L})$. We have computed numerically the spectrum, pseudo-spectrum and Kreiss constant of the linear operator $\mathcal{L}$ using the material parameters for periodically poled lithium niobate $[1,3,12]$. We have also assumed that the pump pulse is a Gaussian of FWHM of 4ps. Typical results are shown in Figure 2 and Table 1. For all the values of $\tau_{c}$ used in this paper the pump amplitude $P_{a}$ has been chosen so that $\lambda_{M} \simeq 0.87$, i.e. the zero signal and idler solution is stable: the asymptotic parametric amplitude gain at each pass is approximately 2.3, not enough to compensate for the losses at the mirrors ( $R=0.37$ for the cases in Figure 2). For a small positive value of $\tau_{c}$ (i.e. the pump repeat time is longer than the cavity round trip time) there is a small transient amplification, by approximately a factor of 20 , but it is not sufficient to allow the formation of noise sustained structures. As can be seen from the panel (A-2) of Figure 2 an initial perturbation of the signal displays transient growth, but ultimately decays to zero. As $\tau_{c}$ becomes negative, the pump value is increased by a factor of almost two in order to keep $\lambda_{M} \simeq 0.87$. At the same time, the transient amplification factor increases from 20 to $5 \cdot 10^{9}$ and a non-zero signal field, entirely noise-driven, develops, even though the zero signal field solution is nominally stable. There is no significant drift in the signal field, indicating that it has the same repetition period of the pump, even though the cavity round-trip time is slightly longer. The pump pulse energy corresponding to this last case is $0.8 \mathrm{~nJ}$ for a 4 ps FWHM pulse focused on an $33 \mu \mathrm{m}$ diameter spot [12], corresponding to $80 \mathrm{~mW}$ power with $100 \mathrm{MHz}$ repetition rate (equivalent to a cavity length of $3 \mathrm{~m}$ ). As can be seen from Figure 2(B-2) the peak intensity of the corresponding signal pulse is approximately nine times that of the pump.

In conclusion, we have shown that SPOPOs are capable of enormous amplification ratios, of the order of $10^{9}$ : in these regimes SPOPOs emit a signal field, even though the zero signal field solution is stable. The emitted signal is entirely noise driven and is expected to be correlated to the photon fluctuations in the cavity. The correct representation of quantum fluctuations in a semiclassical framework [5], like the one used here, is a natural extension of the preliminary results presented in this paper and will be reported elsewhere. Whatever the source of noise, pseudospectra offer a computationally efficient manner to quantify the transient amplification factor and determine the best parameters to observe the remarkable phenomenon of giant noise amplification.

We thank F. Papoff, G.-L. Oppo and A. Scroggie for their help. We were partly supported by a Nuffield Foundation Undergraduate Research Bursary (URB/34177).

\section{References}

1. A. J. Scroggie, G.-L. Oppo, and G. D'Alessandro, "Sensitivity to noise in synchronously pumped optical parametric oscillators," J. Opt. Soc. Am. B 17, 84-89 (2000). 
2. J. D. V. Khaydarov, J. H. Andrews, and K. D. Singer, "Pulse-Compression mechanism in a synchronously pumped optical parametric oscillator," JOSA B 12, 2199-2208 (1995).

3. L. Lefort, K. Puech, S. D. Butterworth, Y. P. Svirko, and D. C. Hanna, "Generation of femtosecond pulses from order-of-magnitude pulse compression in a synchronously pumped optical parametric oscillator based on periodically poled lithium niobate," Opt. Lett. 24, 28-30 (1999).

4. J. Khurgin, J. M. Melkonian, A. Godard, M. Lefebvre, and E. Rosencher, "Passive mode locking of optical parametric oscillators: an efficient technique for generating subpicosecond pulses," Opt. Express 16, 4804-4818 (2008).

5. R. Zambrini, S. M. Barnett, P. Colet, and M. San Miguel, "Macroscopic quantum fluctuations in noise-sustained optical patterns," Phys. Rev. A 65, 023813 (2002).

6. L. N. Trefethen and M. Embree, Spectra and Pseudospectra (Princeton University Press, 2005).

7. A. Haché, G. R. Allan, and H. M. van Driel, "Effects of cavity detuning on the pulse characteristics of a femtosecond synchronously pumped optical parametric oscillator," J. Opt. Soc. Am. B 12, 2209-2213 (1995).

8. F. Papoff, G. D'Alessandro, and G.-L. Oppo, "State Dependent Pseudoresonances and Excess Noise," Phys. Rev. Lett. 100, 123905 (2008).

9. K. Petermann, "Calculated spontaneous emission factor for double-heterostructure injection lasers with gain-induced waveguiding," IEEE J. Quantum Electron. 15, 566-570 (1979).

10. W. J. Firth and A. M. Yao, "Giant Excess Noise and Transient Gain in Misaligned Laser Cavities," Phys. Rev. Lett. 95, 073903 (2005).

11. L. N. Trefethen, A. E. Trefethen, S. C. Reddy, and T. A. Driscoll, "Hydrodynamic stability without eigenvalues," Science 261, 578-585 (1993).

12. S. D. Butterworth, V. Pruneri, and D. C. Hanna, "Optical parametric oscillation in periodically poled lithium niobate based on continuous-wave synchronous pumping at 1.047 mu m," Opt. Lett. 21, 1345 (1996). 


\begin{tabular}{|l|l|l||l|l|l|}
\hline \multicolumn{2}{|c||}{$P_{a}=2.0 \& \tau_{c}=0.41 \mathrm{ps}$} & \multicolumn{3}{c|}{$P_{a}=3.6 \& \tau_{c}=-0.96 \mathrm{ps}$} \\
\hline \hline $\log _{10}(\epsilon)$ & $\rho_{\epsilon}(\mathcal{L})$ & $\mathcal{K}_{\epsilon}$ & $\log _{10}(\epsilon)$ & $\rho_{\epsilon}(\mathcal{L})$ & $\mathcal{K}_{\epsilon}$ \\
\hline-1 & 1.29 & 2.9 & -9 & 1.26 & $2.6 \cdot 10^{8}$ \\
-2 & 1.11 & 11 & -10 & 1.14 & $1.4 \cdot 10^{9}$ \\
-3 & 1.02 & 20 & -11 & 1.05 & $5 \cdot 10^{9}$ \\
\hline \multicolumn{3}{|c||}{$\mathcal{K} \gtrsim 20$} & \multicolumn{3}{c|}{$\mathcal{K} \gtrsim 5 \cdot 10^{9}$} \\
\hline \hline
\end{tabular}

Table 1. Estimate of Kreiss constant for the two examples shown in Figure 2. 


\section{List of Figures}

1 The noisy leading edge of the signal pulse (dashed line) arrives at the crystal when the pump pulse (solid line) is very large and is amplified together with the idler (dot-dashed line). Losses during propagation quench the nonamplified tail of the signal pulse. . . . . . . . . . . . . . . . . . .

2 Numerical pseudo-spectra of $\mathcal{L}$ and corresponding evolution of the signal field for two values of the pump amplitude $P_{a}$ and detuning $\tau_{c}$. (A-1) and (B-1) show the eigenvalues of $\mathcal{L}$ (dots), the unit circle (thick circle) and boundaries of $\Lambda_{\epsilon}(\mathcal{L})$ for $\epsilon=\left\{10^{0}, \ldots, 10^{-10}\right\}$ in the complex plane. (A-2) and (B-2) show the corresponding signal amplitude, obtained by numerically integrating eq. (1), as a function of round trip and $\tau$. The (dimensionless) parameter values correspond to those for lithium niobate $[1,3]: v_{j}^{-1}=\{1.0166,1,1.0049\}$, $\beta_{j}=\{-1.29,-0.343,1.47\} \times 10^{-7}, \rho_{j}=\{0,0,0\}, \Delta k=0, \theta=0, R=0.37$, $\tau_{p}=0.0244$. A $\delta$-correlated noise with amplitude $A_{j}=10^{-8}$ was added to the equations. One unit of dimensionless time is equivalent to $137 \mathrm{ps}$ and the length of the crystal is $20 \mathrm{~mm}$. . . . . . . . . . . . . . . . 


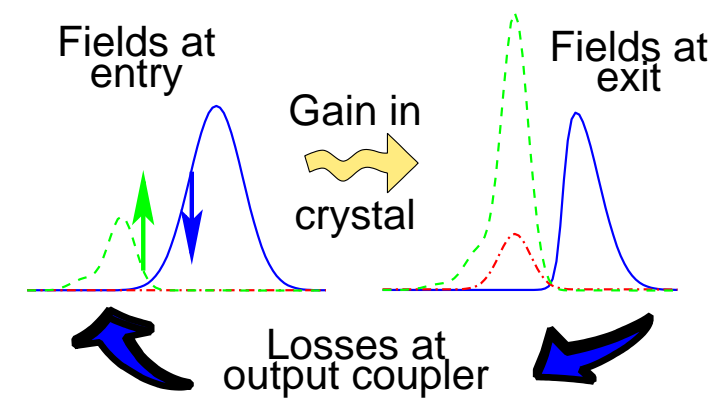

Fig. 1. The noisy leading edge of the signal pulse (dashed line) arrives at the crystal when the pump pulse (solid line) is very large and is amplified together with the idler (dot-dashed line). Losses during propagation quench the non-amplified tail of the signal pulse. 

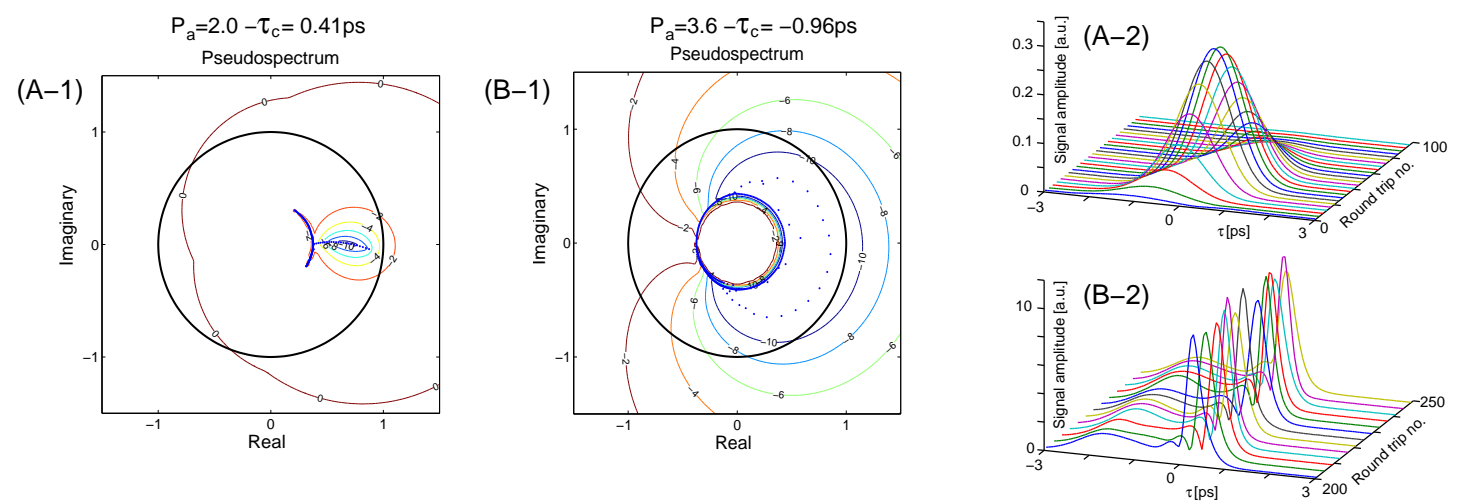

Fig. 2. Numerical pseudo-spectra of $\mathcal{L}$ and corresponding evolution of the signal field for two values of the pump amplitude $P_{a}$ and detuning $\tau_{c}$. (A-1) and (B-1) show the eigenvalues of $\mathcal{L}$ (dots), the unit circle (thick circle) and boundaries of $\Lambda_{\epsilon}(\mathcal{L})$ for $\epsilon=\left\{10^{0}, \ldots, 10^{-10}\right\}$ in the complex plane. (A-2) and (B-2) show the corresponding signal amplitude, obtained by numerically integrating eq. (1), as a function of round trip and $\tau$. The (dimensionless) parameter values correspond to those for lithium niobate $[1,3]: v_{j}^{-1}=\{1.0166,1,1.0049\}$, $\beta_{j}=\{-1.29,-0.343,1.47\} \times 10^{-7}, \rho_{j}=\{0,0,0\}, \Delta k=0, \theta=0, R=0.37, \tau_{p}=0.0244$. A $\delta$-correlated noise with amplitude $A_{j}=10^{-8}$ was added to the equations. One unit of dimensionless time is equivalent to $137 \mathrm{ps}$ and the length of the crystal is $20 \mathrm{~mm}$. 